Case Report

\title{
Massive Ascites in a Renal Transplant Patient after Laparoscopic Fenestration of a Lymphocele
}

\author{
Shohei Kawaguchi, ${ }^{1}$ Takahiro Nohara, ${ }^{2}$ Takashi Shima, \\ Satoko Matsuyama, ${ }^{2}$ Chikako Nose, ${ }^{3}$ Junya Yamahana, ${ }^{3}$ Yoshifumi Kadono, \\ Chikashi Seto, ${ }^{1}$ Masahiko Kawabata, ${ }^{3}$ and Atsushi Mizokami ${ }^{2}$ \\ ${ }^{1}$ Department of Urology, Toyama Prefectural Central Hospital, Toyama, Japan \\ ${ }^{2}$ Department of Integrative Cancer Therapy and Urology, Kanazawa University Graduate School of Medical Science, Kanazawa, Japan \\ ${ }^{3}$ Department of Internal Medicine, Toyama Prefectural Central Hospital, Toyama, Japan \\ Correspondence should be addressed to Shohei Kawaguchi; shohei_k2001@yahoo.co.jp
}

Received 11 August 2016; Accepted 19 October 2016

Academic Editor: Marian Klinger

Copyright (c) 2016 Shohei Kawaguchi et al. This is an open access article distributed under the Creative Commons Attribution License, which permits unrestricted use, distribution, and reproduction in any medium, provided the original work is properly cited.

\begin{abstract}
Retroperitoneal lymphocele is a common complication of renal transplantation. Here, we report the case of a 67-year-old woman with massive ascites after fenestration surgery for a lymphocele that developed following renal transplantation. She had been on continuous ambulatory peritoneal dialysis for 9 years. Living donor renal transplantation was performed and an intrapelvic lymphocele subsequently developed. The lymphocele did not resolve after aspiration therapy; therefore, laparoscopic fenestration was performed. Although the lymphocele disappeared, massive ascites appeared in its stead. Half a year later, the ascites was surgically punctured, which then gradually resolved and disappeared 6 weeks later. Aspiration therapy should be considered in patients on long-term peritoneal dialysis, although laparoscopic fenestration is safe and effective.
\end{abstract}

\section{Introduction}

Retroperitoneal lymphocele is a common complication after renal transplantation. The incidence rate of lymphocele after renal transplantation is $1 \%-26 \%$ [1]. The lymphocele is usually small, asymptomatic, and incidentally diagnosed during routine ultrasonography. However, symptomatic lymphoceles may affect graft function and thus require treatment. The treatment options for lymphoceles occurring after renal transplantation include simple aspiration, sclerotherapy, and drain placement. Laparoscopic and open peritoneal fenestration surgeries are more invasive options. Previous studies found that laparoscopic fenestration is a safe, effective, and possible first-line treatment for larger, symptomatic lymphoceles when there is a high risk for graft dysfunction $[2,3]$.

To the best of our knowledge, this is the first report of massive ascites developing in a renal transplant patient after peritoneal fenestration surgery for a symptomatic lymphocele.

\section{Case Presentation}

A 67-year-old woman who had been on continuous ambulatory peritoneal dialysis (CAPD) for 9 years received a living donor renal transplant. There was no history of peritonitis during CAPD. Graft function was good and her serum creatinine level was $0.9 \mathrm{mg} / \mathrm{dL} 5$ days after the transplantation. The patient received combination immunosuppressive therapy with methylprednisolone, tacrolimus, mycophenolate mofetil, and basiliximab.

Perirenal fluid collection, detected 8 days after transplantation during routine ultrasonography, increased on a daily basis. On day 14, simple aspiration was performed as lower abdominal pressure was increasing. The creatinine level of the drainage was equivalent to that of the serum. After aspiration, fluid immediately reaccumulated in the perirenal space (Figure 1(a)). On day 32, laparoscopic surgery to fenestrate the lymphocele into the peritoneal cavity was performed by introducing a $\sim 5 \mathrm{~cm}$ incision in the peritoneum. The septa of the lymphocele were then removed. 


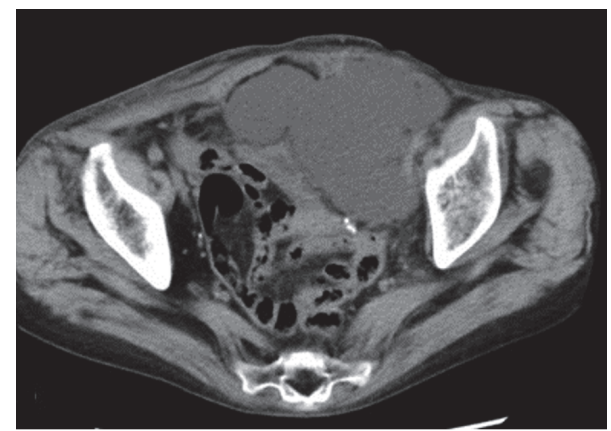

(a)

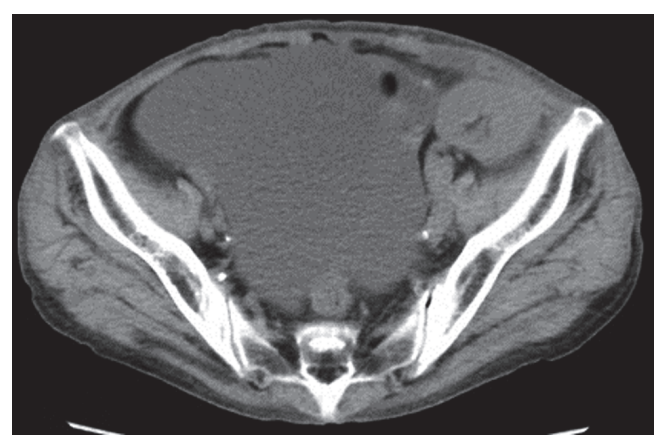

(b)

Figure 1: The lymphocele developed from around the graft to the front of the bladder after transplantation (a). The ascites developed and increased after fenestration surgery (b).

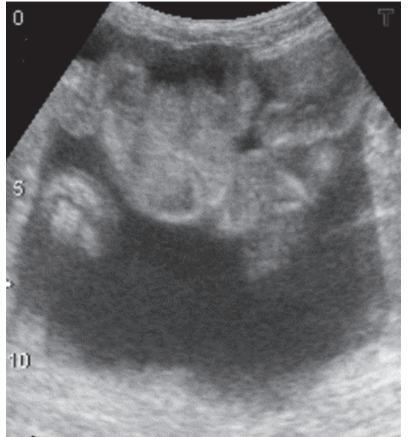

(a)

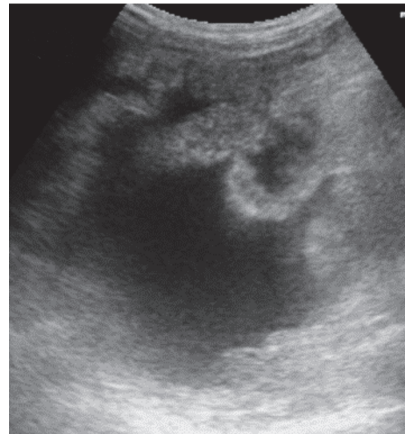

(b)

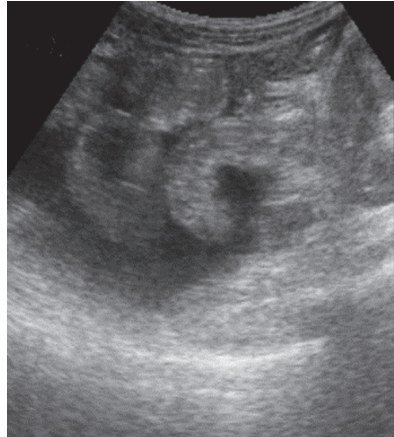

(c)

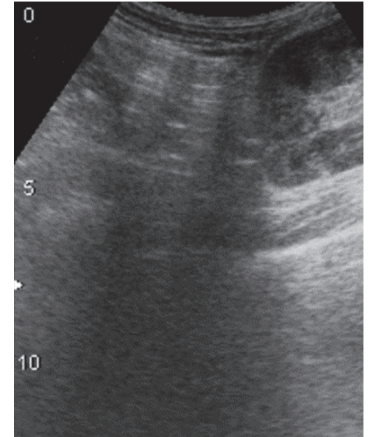

(d)

FIGURE 2: Lower abdominal ultrasonography. (a) Before percutaneous abdominal puncture; (b) 2 weeks after abdominal puncture; (c) 4 weeks after abdominal puncture; and (d) 6 weeks after abdominal puncture (ascites completely resolved).

Three days after fenestration, the lymphocele had almost completely resolved. However, ascites was detected with ultrasonography 5 days after fenestration, which gradually increased. On day 79, abdominal computed tomography was performed because of intense abdominal fullness (Figure 1(b)). Massive ascites was detected and a diagnostic puncture was performed. Laboratory analysis of the fluid revealed the following characteristics: specific gravity (1.017), total protein concentration $(2.8 \mathrm{~g} / \mathrm{dL}$ ), Rivalta reaction (negative), and ratio of ascites to serum lactate dehydrogenase (0.5). Culture and cytodiagnosis of the ascites and a T-Spot tuberculosis assay were all negative. The patient was followed up as an outpatient because the ascites was transudative and not cancerous or tuberculous.

On day 163 after fenestration, the patient presented to the outpatient clinic with prominent abdominal fullness. A percutaneous abdominal puncture was performed and $2 \mathrm{~L}$ of ascitic fluid was aspirated. Two weeks after aspiration, the ascites significantly decreased and the abdominal fullness was resolving. The ascites continued to decrease and disappeared 6 weeks after aspiration (Figure 2). There was no recurrence after 2.5 years of follow-up.

\section{Discussion}

Lymphocele after renal transplantation results from transection of the lymphatic vessels accompanying the external iliac vessels during transplantation surgery and subsequent lymph accumulation in a nonepithelized retroperitoneal cavity. Dissection around the external iliac vessels is necessary to perform vascular anastomoses. However, extensive dissection is a risk factor for lymphocele formation [4]. Limited dissection and careful ligation of iliac lymphatic vessels decrease the incidence rate of lymphoceles [5].

Management of lymphocele after renal transplantation is variable. Aspiration therapy is performed to reduce leakage of lymphatic fluid, and fenestration surgery is performed to allow the peritoneum to absorb the fluid. Laparoscopic surgery to fenestrate the lymphocele into the peritoneal cavity is preferred because the rate of recurrence ( $8 \%)$ is lower than that with open surgery (16\%) and aspiration therapy (59\%) [1].

Long-term CAPD can lead to structural changes in the peritoneal membrane, such as loss of mesothelial cells, thickening of the submesothelial zone, and alterations of blood vessel walls [6]. Functional transport abnormalities of the peritoneal membrane, such as loss of ultrafiltration, occur subsequent to these structural changes [7].

In the present case, we performed laparoscopic fenestration after simple aspiration. Although the lymphocele disappeared, a large quantity of ascites developed. Nine years of CAPD may have produced structural changes in and loss of function of the peritoneal membrane. A massive amount of lymph fluid then leaked from around the external iliac vessels 
and accumulated in the peritoneal cavity without absorption. A percutaneous abdominal puncture was performed 5 months after fenestration, and the ascites subsequently decreased and disappeared 6 weeks after aspiration. The resolution of the ascites may have been caused by the decrease in lymph fluid leakage due to long-term high abdominal pressure.

Laparoscopic fenestration is a safe and effective first-line treatment for symptomatic lymphocele occurring after renal transplantation. However, aspiration therapy, such as percutaneous catheter drainage with or without sclerotherapy, should be considered for patients with peritoneal function that is impaired by long-term peritoneal dialysis.

\section{Competing Interests}

The authors declare that they have no competing interests.

\section{Acknowledgments}

The authors would like to thank Enago (https://www.enago $. j \mathrm{p} /$ ) for the English language review.

\section{References}

[1] A. Lucewicz, G. Wong, V. W. T. Lam et al., "Management of primary symptomatic lymphocele after kidney transplantation: a systematic review," Transplantation, vol. 92, no. 6, pp. 663-673, 2011.

[2] H. S. Lee, D. J. Joo, K. H. Huh et al., "Laparoscopic fenestration versus percutaneous catheter drainage for lymphocele treatment after kidney transplantation," Transplantation Proceedings, vol. 45, no. 4, pp. 1667-1670, 2013.

[3] F. Ulrich, S. Niedzwiecki, P. Fikatas et al., "Symptomatic lymphoceles after kidney transplantation-multivariate analysis of risk factors and outcome after laparoscopic fenestration," Clinical Transplantation, vol. 24, no. 2, pp. 273-280, 2010.

[4] E. Zagdoun, M. Ficheux, T. Lobbedez et al., "Complicated lymphoceles after kidney transplantation," Transplantation Proceedings, vol. 42, no. 10, pp. 4322-4325, 2010.

[5] O. Øyen, V. Siwach, P.-D. Line et al., "Improvement of posttransplant lymphocele treatment in the laparoscopic era," Transplant International, vol. 15, no. 8, pp. 406-410, 2002.

[6] I. Savidaki, D. Karavias, F. Sotsiou et al., "Histologic change of peritoneal membrane in relation to adequacy of dialysis in continuous ambulatory peritoneal dialysis patients," Peritoneal Dialysis International, vol. 23, no. 2, pp. S26-S30, 2003.

[7] R. T. Krediet, "The peritoneal membrane in chronic peritoneal dialysis," Kidney International, vol. 55, no. 1, pp. 341-356, 1999. 


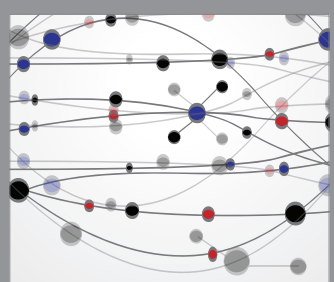

The Scientific World Journal
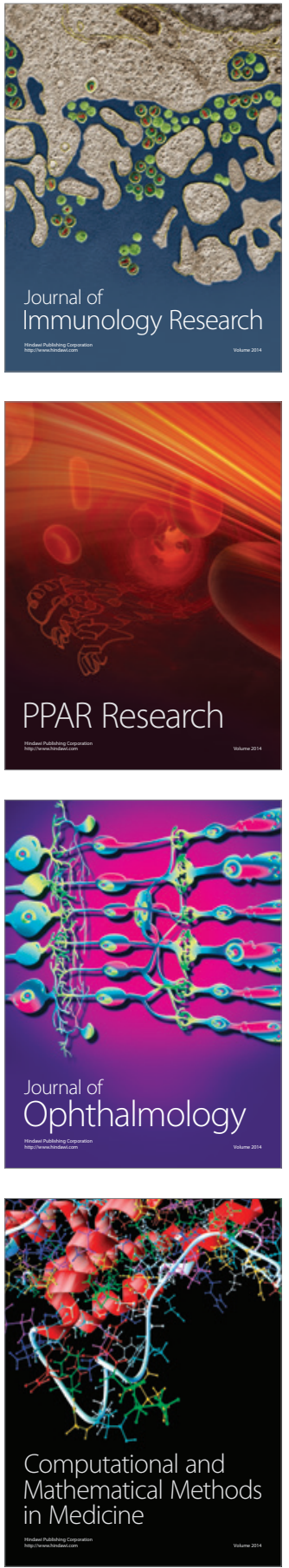

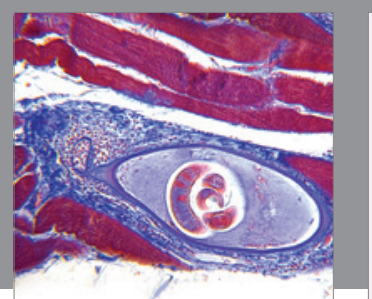

Gastroenterology Research and Practice

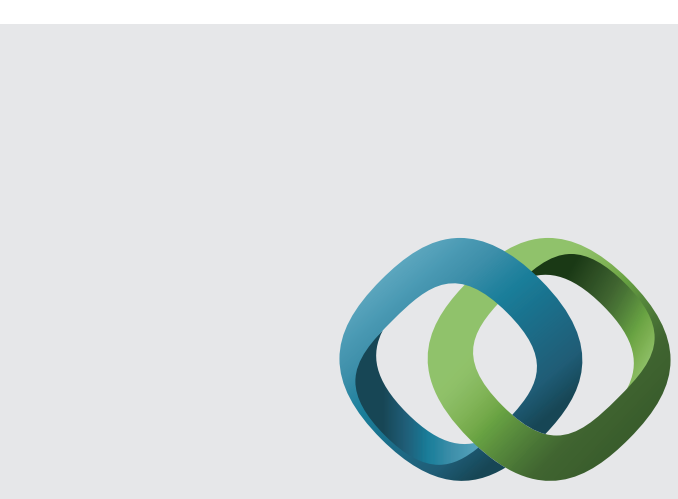

\section{Hindawi}

Submit your manuscripts at

http://www.hindawi.com
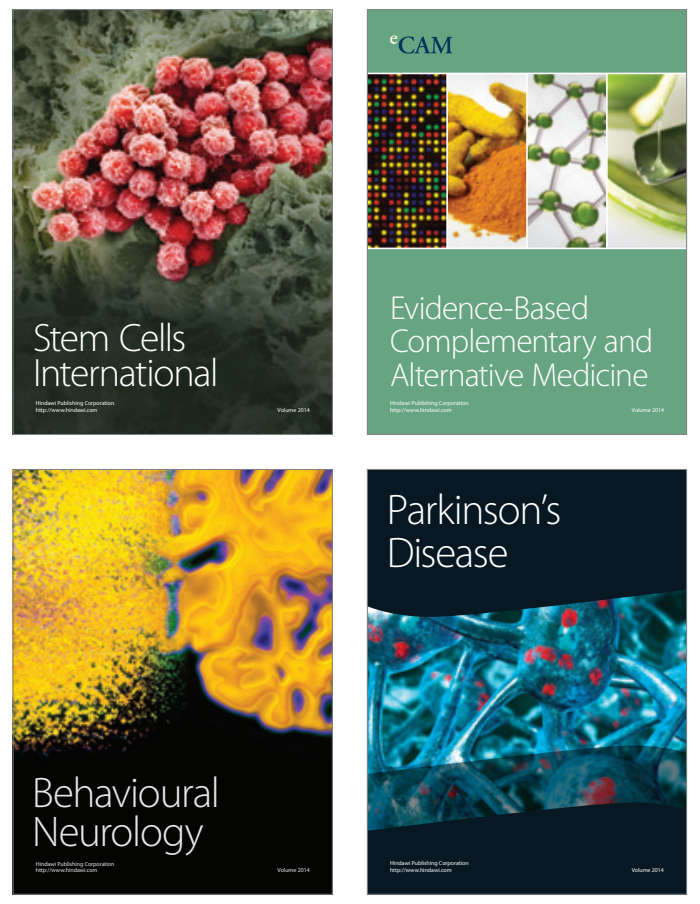
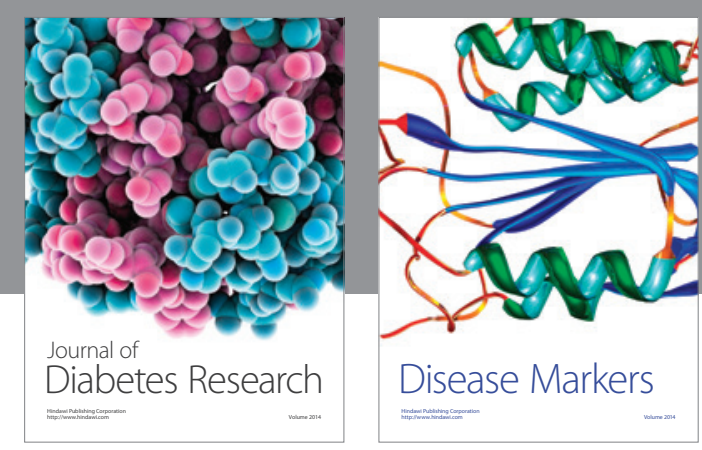

Disease Markers
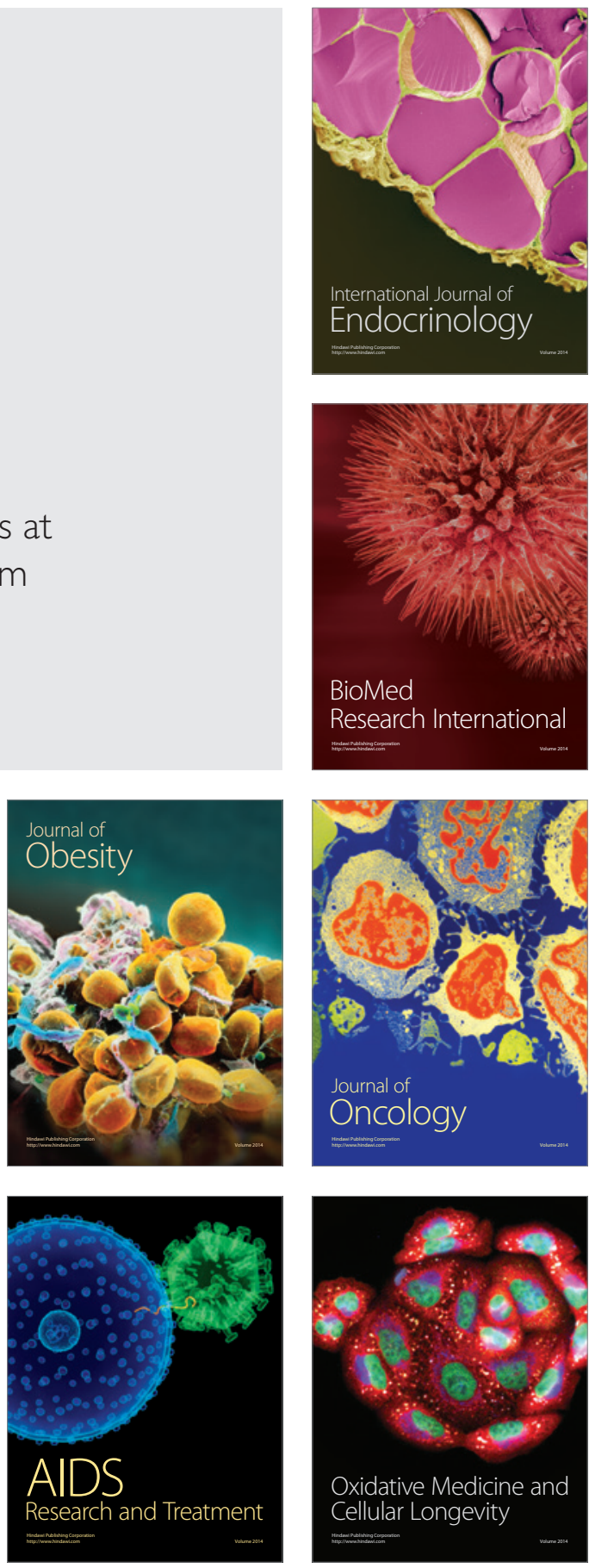